\title{
RESOLUTION I
}

IAU Colloquium No. 56, "Reference Coordinate Systems for Earth Dynamics,"

Recognizing:

1) That geodynamics has become the subject of intensive international research during the last decade,

2) That a common requirement for all investigations is the necessity of a well-defined terrestrial coordinate system not available at present,

3) That the use of new techniques, such as Lageos laser ranging, LLR, VLBI, astrometric satellites, when used in a well-coordinated manner can determine and maintain such a system,

\section{Recommends:}

That a working group be established by the Presidents of IAU Commissions 4, 19, 31, and the President of IAG to prepare a proposal for the establishment and maintenance of a Conventional Terrestrial Reference System. This system is to include provisions for the replacement of the presently used terrestrial reference frame (such as the one partially defined by the CI0 and the BIH zero meridian), providing continuity, and conformance with the IAU 1976 and 1979 resolutions regarding astronomical constants and the theory of nutation, or its possible modification, as well as with the IUGG Geodetic Reference System 1980.

The colloquium further recommends that the working group should report its findings for discussion and possible adoption at the 1982/83 assemblies of the IAU and the IUGG/IAG. 